\title{
Alcohol consumption is associated with structural changes in various organ systems: A population-based study in UK Biobank
}

Evangelos Evangelou ${ }^{1,2}$, Hideaki Suzuki ${ }^{3,45^{*}}$, Wenjia Bai ${ }^{5,6^{*}}$, Raha Pazoki $^{7,8}$, He Gao ${ }^{1}$, Paul M Matthews $^{5,9}$, Paul Elliott ${ }^{1,7,9}$,

1.Department of Epidemiology and Biostatistics, School of Public Health, Imperial College London, London, UK

2.Department of Hygiene and Epidemiology, University of Ioannina Medical School, Ioannina, Greece

3.Department of Cardiovascular Medicine, Tohoku University Hospital, Japan

4.Tohoku Medical Megabank Organization, Tohoku University, Japan

5.Department of Brain Sciences, Imperial College London, London, UK

6.Data Science Institute, Imperial College London, London, UK

7.MRC-PHE Centre of Environment and Health, School of Public Health, Imperial College London, London, UK

8.Division of Biomedical Sciences, Department of Life Sciences, College of Health and Life Sciences, Brunel University London, London, UK

9. UK Dementia Research Institute at Imperial College London, London, UK

* equal contribution

Corresponce to:

Professor Paul Elliott MBBS PhD FRCP FFPH FMedSci

Professor of Epidemiology and Public Health Medicine

Head of Department

Director, MRC-PHE Centre for Environment and Health

Department of Epidemiology and Biostatistics

School of Public Health, Faculty of Medicine

Imperial College London

St Mary's Campus

Norfolk Place, LONDON, W2 1PG

Tel: +44(0)2075943328

p.elliott@imperial.ac.uk

www.imperial.ac.uk 


\begin{abstract}
Excessive alcohol consumption is associated with damage to various organs, but its multiorgan effects have not been characterised across the usual range of alcohol drinking in a large general population sample. We assessed global effects of alcohol consumption on quantitative magnetic resonance imaging phenotypic measures of the brain, heart, aorta and liver of UK-Biobank participants who reported drinking alcohol. We found a monotonic association of higher alcohol consumption with lower normalised brain volume across the range of alcohol intakes $\left(-1.7 \times 10^{-3} \pm 0.76 \times 10^{-3}\right.$ per doubling of alcohol consumption, $\left.P=3.0 \times 10^{-14}\right)$. Alcohol consumption also was associated directly with measures of left ventricular mass index and left ventricular and atrial volume indices. Liver fat increased by a mean of $0.15 \%$ per doubling of alcohol consumption. Our results imply that there is not a "safe threshold" below which there are no toxic effects of alcohol. Current public health guidelines concerning alcohol consumption may need to be revisited.
\end{abstract}




\section{Introduction}

Alcohol consumption causes damage to multiple organs and systems and heavy drinking is associated with increased all-cause mortality(1). According to the Global Burden of Diseases

Study, alcohol use was the seventh leading risk factor for both deaths and Disability-Adjusted

Life Year (DALYs) in 2016, accounting for $2.2 \%$ and $6.8 \%$ excess in age standardized

female and male deaths, respectively(2). While previous evidence has suggested that low to

moderate amounts of daily consumption may have beneficial effects on cardiovascular

health(1), a recent large-scale meta-analysis concluded that even moderate daily alcohol

intake may have significant impact on disease risk(3). Because of these uncertainties, there

remains controversy about whether there is a "safe level" of alcohol drinking for the general

population $(4,5)$

The liver is a primary target for the detrimental effects of alcohol, as it is the primary site of

alcohol metabolism(6). With high levels of alcohol consumption, effects on other organs

(including the brain and heart) have been described(7). Excessive alcohol use during

adolescence has been associated with reduced brain grey matter volumes(8), but evidence

regarding structural brain changes at lower levels of alcohol intake is limited and

conflicting(9-11). Moderate to heavy alcohol consumption is implicated causally with

pathologically reduced left ventricular ejection fraction(12), cardiomyopathy, heart failure

and sudden death. Analyses of cardiac structure based on echocardiography have suggested 
that smaller differences in left ventricular mass consistent with early pathology can also be attributed to lower levels of alcohol intake(13-15)

Here, for the first time, we report associations across the range of population alcohol consumption with differences in morphology or function of multiple organs determined from quantitative measures of the brain, cardiac structure and function and liver fat Magnetic Resonance Imaging (MRI) scans. Our aim was to investigate effects of alcohol at intakes within the currently recommended limits for consumption by the general population.

Discovery of evidence for potentially toxic effects of alcohol within these recommended limits would have important implications for public health and government policies regarding "safe" levels of alcohol drinking.

\section{Methods}

\section{Study participants}

UK Biobank is a prospective, observational study of $\sim 500,000$ people from across the United

Kingdom, aged 40-69 years at recruitment $(2007-2010)(16,17)$. Here we used a subset of the

UK Biobank data from participants whose brain, cardiac and/or aortic and liver MRI images

and image derived phenotypes (IDPs) were available. Non-drinkers and those with self-

reported brain, cardiac and/or aortic diseases were excluded. IDPs of participants were 
included based on availability of measures after application of exclusion criteria (brain grey

matter [N=10,143], brain white matter $[\mathrm{N}=9,053]$, heart $[\mathrm{N}=11,822]$, aortic $[\mathrm{N}=12,376]$ and

liver [N=3,649]) ( Supplementary Figure 1). Table 1 describes characteristics of the

population included in the analyses. The study is reported following the Strengthening the

Reporting of Observational Studies in Epidemiology (STROBE) guideline.

\section{Baseline characteristics}

Information on age, sex, ethnicity, college degree education, body mass index (BMI),

hypertension, diabetes, and history of smoking and cardiac, brain and/or aortic diseases were

reported at the imaging assessment. We defined participants as hypertensive if they had

systolic $\geq 140$ or diastolic blood pressure $\geq 90 \mathrm{mmHg}$ or were receiving antihypertensive

medication(18). We recorded self-reported diabetes, smoking history, and college degree

education.

\section{Alcohol consumption}

We calculated alcohol intake as grams of alcohol per day (g/d) among drinkers based on self-

reported alcohol drinking from a touch-screen questionnaire described previously(19).

Briefly, the quantity of each type of drink was multiplied by its standard drink size and

reference alcohol content. Drink-specific intake during the reported drinking period was 
summed and converted to $\mathrm{g} / \mathrm{d}$ alcohol intake for each participant with completed responses to the quantitative drinking questions. The alcohol intake for participants with incomplete responses was imputed by bootstrap resampling from the completed responses, stratified by drinking frequency and sex. Alcohol intake was $\log _{2}$-transformed, as it has a skewed distribution. Using this transformation, a $\log _{2}$ change of 1 unit translates to a doubling of alcohol consumption, e.g. from $10 \mathrm{~g} /$ day to $20 \mathrm{~g} /$ day.

\section{Brain MRI acquisition and pre-processing}

Details of the image acquisition are available online(20). Briefly, the T1-weighted (3D

MPRAGE, $1 \times 1 \times 1 \mathrm{~mm}^{3}$ resolution, field of view $(\mathrm{FOV}) /$ matrix $=208 \times 256 \times 256$, TR $($ repetition time $)=2000 \mathrm{~ms}$, TI (inversion time $)=880 \mathrm{~ms}$ ) brain images used here were acquired using a Siemens Skyra 3T running VD13A SP4 (Siemens Healthcare, Erlangen, Germany) with a Siemens 32-channel RF receive head coil were used for structural analyses. Before analyses, the images were registered in the standard Montreal Neurological Institute (MNI) space using DARTEL tools in SPM12 (https://www.fil.ion.ucl.ac.uk/spm/software/spm12/).

After separate segmentation of grey and white matter and cerebrospinal fluid, each tissue mask was modulated with the Jacobian determinants derived from the spatial normalisation, multiplying each voxel by the relative change in volume to correct for volume changes in the non-linear normalisation(21). Brain and regional volumes were normalised to the 
corresponding total intracranial volumes, calculated from the sums of volumes of the grey

and white matter and cerebrospinal fluid. For the multiple regression voxel-wise analysis, the normalized grey matter maps were smoothed by convolving an isotropic Gaussian kernel of 8-mm full width at half maximum, excluding voxels with a grey matter probability value < 0.2 .

Brain diffusion MRI images were acquired using a Stejskal-Tanner pulse sequence(22). Our analyses used the white matter microstructural IDPs for fractional anisotropy and orientation dispersion (the extent of directional complexity of diffusion) ${ }^{3,(23)}$ for 27 probabilistically defined(18) white matter tracts described and made available in the UK Biobank Data Showcase(24). The white matter microstructure measures were then expressed as mean zscores (referenced to the mean values for the full study population) in our analyses.

\section{Cardiac and aortic MRI acquisition and pre-processing}

Details of the cardiac and aortic image acquisitions were reported previously(25). Briefly, the. cardiac and aortic MRI were acquired using a clinical wide bore 1.5T scanner (MAGNETOM Aera, Syngo Platform VD13A, Siemens Healthcare, Erlangen, Germany) with 48 receiver channels, a $45 \mathrm{mT} / \mathrm{m}$ and $200 \mathrm{~T} / \mathrm{m} / \mathrm{s}$ gradient system and an 18-channel anterior body surface coil used in combination with 12 elements of an integrated 32 element spine coil and electrocardiographic gating for synchronization with the cardiac cycle. The 
acquired images were segmented to derive IDPs using a fully convolutional network

$(\mathrm{CNN})(26,27)$.

The ventricular CNN image segmentation provided measures that, with adjustments for body

surface area, were used as IDPs for the left ventricular mass, left end-diastolic (LVEDVI) and

end-systolic volume (LVESVI) and right end-diastolic (RVEDVI) and right end-systolic

volume (RVESVI) indices. Left and right ventricular ejection fraction IDPs were derived

from integrations of the primary indices as (LVEDVI-LVESVI)/LVEDVI $\times 100$ and

(RVEDVI-RVESVI)/RVEDVI $\times 100$, respectively. The atrial image segmentation provided

left and right atrial volume indices after adjustment for body surface area. The aortic image

segmentation provided maximal ascending (AAoAI) and descending (DAoAI) aortic area

indices and minimal ascending $\left(\mathrm{AAoAI}_{\min }\right)$ and descending $\left(\mathrm{DAoAI} \mathrm{min}_{\mathrm{in}}\right)$ aortic area indices

after adjustment for body surface area. Ascending and descending aortic distensibilities were

derived as $\left(\mathrm{AAoAI}-\mathrm{AAoAI} \mathrm{min}_{\mathrm{n}}\right) / \mathrm{AAoAI}_{\min } /($ systolic - diastolic blood pressure) and (DAoAI-

$\left.\mathrm{DAoAI}_{\mathrm{min}}\right) / \mathrm{AAoAI}_{\mathrm{min}} /($ systolic - diastolic blood pressure), respectively(25).

\section{Liver fat MRI acquisition and pre-processing}

Abdominal images for assessments of liver fat were acquired using a Siemens 1.5T

MAGNETOM Aera. Details of the MRI acquisition and pre-processing protocol are provided

elsewhere(28). Briefly, a dual-echo Dixon Vibe protocol, which can be used to generate 
images distinguishing water and fat, from which liver fat could be determined, was

performed $(2.2 \times 1.2 \times 10 \mathrm{~mm} 3$ resolution, $\mathrm{TR}=3.23 \mathrm{~ms}, \mathrm{TE}=1.44 \mathrm{~ms})$. The liver MRI proton density fat fraction \% derived is available to researchers through the UK Biobank Data Showcase(24).

\section{Statistical analysis}

We estimated the age-related differences in the brain normalised volume, cardiac and liver fat

IDPs by their regression onto age adjusted for sex, ethnicity, educational level, BMI,

hypertension, diabetes, and smoking history. We then examined the magnitudes of

differences in organ morphology or functional IDPs with alcohol consumption. Each IDP was

regressed onto $\log _{2}$-transformed alcohol consumption adjusted for age, sex, ethnicity,

educational level, BMI, hypertension, diabetes, and smoking history; raw coefficients are

used for all measures except brain white matter diffusion measures, for which standardised

coefficients are reported. Normality of the IDPs was tested using a Shapiro-Wilks test. We

used partial residual plots to assess any deviation from the fitted model. Additionally, a

voxel-wise parametric analysis, ${ }^{4,11}$ which used each voxel of the grey matter maps as

dependent variable and $\log _{2}$-transformed alcohol consumption as independent variable

adjusted for intracranial volume and the same covariates as above, was conducted for

mapping grey matter regions associated with alcohol consumption. In secondary analysis of 
brain, cardiac and liver phenotypes, an interaction term for age and $\log _{2}$ alcohol consumption was included in the regression models. To correct for multiple comparisons the significance level was set to $P<0.017$ and $P<4.5 \times 10^{-3}$ for brain and heart/aorta imaging IDPs respectively whereas for liver fat at $P<0.05$. For the voxel-wise analysis, a family-wise error-corrected threshold of $P<0.05$ was used for grey matter analysis. All statistical analyses were carried out using STATA 14.

Patients involvement: The performed analyses are based on existing data from a populationbased cohort in the UK (UK Biobank). No patients were explicitly engaged in designing the present research question or the outcome measures, nor were they involved in developing plans for recruitment, design, or implementation of the study. No patients were asked to advise on interpretation or writing up of results. Results from UK Biobank are routinely disseminated to study participants via the study website and social media outlets.

\section{Results}

\section{Participant characteristics and imaging phenotypes}

Baseline characteristics of the participants included in this study and summary IDPs are shown in Tables 1 and 2 . For the 5 subsets in our analysis, median alcohol intakes among these drinkers were similar; $20.9 \mathrm{~g} / \mathrm{d}$ (i.e., just over two $10 \mathrm{~g}$ drinking units, where $125 \mathrm{ml}$ of $12.5 \%$ wine is 1.25 drinking units) for men and $\sim 10.7 \mathrm{~g} / \mathrm{d}$ for women with $25^{\text {th }}$ and $75^{\text {th }}$ 
centiles $~ 10.3 \mathrm{~g} / \mathrm{d}$ and $35.8 \mathrm{~g} / \mathrm{d}$ for men and $\sim 3.6 \mathrm{~g} / \mathrm{d}$ and $17.9 \mathrm{~g} / \mathrm{d}$ for women (

\section{Supplementary Table 1).}

\section{Associations of alcohol consumption with brain structure}

Age-related differences in normalised brain volumes (NBV) in the population, adjusted for alcohol consumption, were about $0.3 \%$ lower/year (mean \pm standard error: $-3.0 \times 10^{-3} \pm 0.05$

$\times 10^{-5} /$ year, $\left.\mathrm{P}<1.0 \times 10^{-300}\right)$, consistent with previous studies(29). The contribution of alcohol to the observed brain volume differences was about $0.17 \%$ lower NBV per doubling of alcohol consumption $\left(-1.7 \times 10^{-3} \pm 2.3 \times 10^{-4}, P=3.0 \times 10^{-14}\right)$. Lower volumes per doubling of alcohol consumption of both total grey $\left(-1.2 \times 10^{-3} \pm 1.7 \times 10^{-4}, P=1.9 \times 10^{-12}\right)$ and white $\left(-5.1 \times 10^{-4} \pm 1.2 \times 10^{-4}, P=2.1 \times 10^{-5}\right)$ matter jointly account for the lower brain volumes associated with greater alcohol intake. Partial residual plots confirmed the observed relationship without any deviations from the fitted model (Supplementary Figures 2a-c).

Exploration of voxel-wise parametric associations of the $\log _{2}$-transformed g/d alcohol intake with brain grey matter showed greatest negative associations with regions in the cingulate and orbital frontal cortices, the bilateral insula and thalami (Figure 1). There were no positive correlations between alcohol and grey matter volumes for any of the brain regions after family-wise error correction. 
Finally, given the associations of greater alcohol intake with lower white matter volumes, we explored alcohol-associated differences in fractional anisotropy, a measure of white matter microstructure, across 27 major white matter tract IDPs. The bilateral corticospinal tracts showed increased fractional anisotropy with greater alcohol intake (standardised coefficient, left, $0.013 \pm 0.003$ per doubling of alcohol consumption, $P=1.0 \times 10^{-4}$; right, $0.011 \pm 0.003$, $P=4.20 \times 10^{-4}$ ). This was associated with lower orientation dispersion (standardised coefficient, left, $-0.013 \pm 0.003, P=8.1 \times 10^{-4}$; right, $-0.013 \pm 0.003, P=1.4 \times 10^{-3}$ ), suggesting greater fibre coherence or a relatively reduced density of orthogonally crossing white matter tracts $(23,30)$

\section{Associations of alcohol consumption with heart and aorta}

We first tested for age-related differences in cardiac and aortic IDPs adjusted for alcohol consumption in the population. Left ventricular mass index and the left atrial and left and right ventricular end diastolic volume indices were lower with greater age. Right and left ventricular ejection fractions both were modestly greater with age. There was also a small relative increase in the right atrial volume index with age (Table 3).

$\log _{2}$-transformed alcohol consumption was associated directly with measures of left ventricular and atrial mass and volume. The effects of alcohol on the cardiac IDPs were largely in opposite direction to those for age (Table 3). 
Ascending and descending aortic area indices increased with age, while distensibility

decreased; $\log _{2}$-transformed alcohol consumption associations for the aorta showed the same directions of effect as age (Table3).

The associations with alcohol appeared linear-log with no deviation as indicated by the partial residual plots (Supplementary Figures 1d-n).). We also explored interactions between age and $\log _{2}$ alcohol consumption, which were most evident for the left ventricular mass index and aortic distensibility IDPs ( Supplementary Table 2). There was no evidence for $\mathrm{U}$ shaped associations, i.e., higher values at both the lower and higher ends of the alcohol intake distribution, for any of the aortic or cardiac IDPs.

\section{Association of alcohol consumption with liver fat}

We did not observe age-related differences in liver fat after adjusting for alcohol consumption and other relevant variables $(0.015 \pm 0.01, P=0.14)$. There was an increase of

liver fat per doubling of alcohol consumption $(0.15 \pm 0.06, P=0.006)$, with no deviation from linear-log association observed (Supplementary Figure 1o)

\section{Discussion}

In this large population-based study of the effects of alcohol consumption on different

organs, we found that increasing alcohol intake was associated with reduced brain grey 
matter volume, increased left ventricular mass and volume and aortic area index, reduced descending aortic distensibility and increased liver fat. There was no evidence against a monotonic increase across the range of alcohol intakes, indicating potentially pathological effects of alcohol on the brain, heart and aorta across the full range of alcohol intake in the population, without evidence for a threshold.

Current guidelines for safe alcohol consumption vary between countries, mostly around one or two standard drinks/day. In the UK, the Chief Medical Officers' guideline for both men and women suggests that avoiding more than 14 units/week (corresponding to $16 \mathrm{~g} / \mathrm{day}$ alcohol) on a regular basis maintains health risks at a low level(31). In the US, the suggested threshold is $\leq 2$ drinks/day ( $\sim 28 \mathrm{~g} / \mathrm{d})$ for men and $\leq 1 \mathrm{drink} /$ day $(\sim 14 \mathrm{~g} / \mathrm{d})$ for women(32). In a recent meta-analysis, excess mortality was observed above around $100 \mathrm{~g}$ alcohol intake per week (14.3 g/day), but with reduced incidence of myocardial infarction(3). Our results suggest that alcohol consumption below this threshold and below the currently recommended guidelines worldwide is associated with pathological structural and functional changes in brain, heart, aorta and liver.

Previous research on structural and functional changes in the brain has indicated that excessive alcohol use is associated with abnormal development of the brain grey matter in animals(33) and humans(8), but the studies were small and underpowered. Here we found 
evidence of inverse associations of brain volume with amounts of alcohol consumed in a general population sample of adults. The magnitude of the effects appears to be meaningful: doubling of alcohol consumption (e.g., from 10 to $20 \mathrm{~g}$ per day) was associated with over half the brain volume reduction attributed to a year of aging in the population. This suggests a possible relationship between alcohol consumption and increased susceptibility to age-related brain pathologies and disease, consistent with our recent report of a genetic correlation between alcohol consumption and Alzheimer's disease(19).

The effects of alcohol consumption on the brain appeared to be relatively generalised with a reduction in both white and grey matter volumes with greater alcohol consumption. Alcohol intake has previously been reported to be associated with reduced grey matter volumes in specific areas of the brain including the hippocampus and the inferior-medial frontal and anterior cingulate cortices(34). Our voxel wide-analysis also showed relatively greater associations of alcohol consumption with lower cingulate volumes. and provides new evidence for similar directions of association within the orbital frontal cortex, the bilateral insula and the thalami (Figure 1).

Studies of the effect of alcohol consumption on brain white matter have been less conclusive to date(9-11). This lack of consensus in the literature may in part reflect both study power and the potential complexity of changes in the macroscopic MRI measures of brain 
microstructure(35). Our study, the largest to date, found the ratio of variance to effect was larger for white than grey matter, highlighting the need for larger study sizes to estimate volume differences in white matter. We also found higher fractional anisotropy and decreased orientation dispersion (a measure of greater fibre coherence)(29) in the corticospinal tracts with increasing alcohol consumption. As we have no reason to hypothesise adaptive plasticity with an increased density of descending motor neurons (one interpretation of increased fractional anisotropy)(36), we interpret these observations as reflecting not differences in the structures of corticospinal tracts, but lower densities in major axonal tracts crossing them (e.g., the cingulum bundle and superior longitudinal fasciculus).

This interpretation is broadly consistent with the lower grey matter volumes in associated cortical regions (e.g., the cingulate and anterior prefrontal cortices) reported here and previously with greater alcohol consumption (34). A similar "paradoxical" difference in fractional anisotropy in the context of decreased density of crossing fibre tracts distinguishes people with mild cognitive impairment who progress most rapidly to Alzheimer's disease(37).

We provide evidence for cardiac remodelling including the association of alcohol consumption with larger ventricular masses, end diastolic volumes and left atrial volume indices. Our findings are consistent with previous echocardiographic studies that showed association between increasing alcohol intake and greater left ventricular mass $(14,15,38)$ 
(although a smaller recent MRI study, assessing effects of light-to-moderate alcohol

consumption, reported similar left ventricular mass in drinkers compared to non-

drinkers(13)). Left ventricular mass is a strong prognostic factor for incidence of

cardiovascular disease and mortality(39). Greater atrial indices with higher alcohol

consumption have also been reported(14); atrial enlargement can be considered a risk factor

for several adverse cardiovascular outcomes(40) and is associated with increased risk of heart

failure(41). Alcohol consumption had same directions of effect as aging for the thoracic

aortic measures (larger diameters and lower distensibilities). Such changes may also

contribute to greater risks of cardiovascular disease and mortality $(42,43)$.

A number of studies have suggested a ' $U$ '-shaped association between alcohol drinking and cardiovascular outcomes, even after exclusion of ex-drinkers from the non-drinker

category(44). Our diagnostic plots for the relationship between alcohol and cardiovascular

measures did not provide evidence of deviation from a monotonic association and therefore

did not support a 'U'-shaped association for these measures. Rather, our results point to

pathological effects of regular alcohol intake on the heart and major vessels occurring below

current consumption guidelines.

Excessive alcohol intake is a well-known risk factor for increased liver fat(45), but the

evidence regarding low-to-moderate alcohol consumption has been inconclusive. Several 
prospective studies have reported a lower prevalence or risk of fatty liver(46-48) for low-tomoderate compared to excessive alcohol consumption. However, a randomized trial showed that even moderate consumption of red wine for three months increases liver fat $(49,50)$. Our results are consistent with this. The magnitude of the association with alcohol $(0.15 \%$ per doubling of alcohol consumption) suggests that alcohol could explain a major proportion of the population variance in liver fat (mean liver fat density percentages in a larger UK Biobank sample ranged between a mean of 1.34- 5.71\%)(51).

Our results highlight the multi-organ effects of low and moderate levels of alcohol consumption in late middle-aged people. This may reflect direct toxicities. For example, the toxic effect of alcohol or its metabolites can cause myocardial damage that leads to increased rates of cardiomyopathies, heart failure and mortality $(3,52)$. Deleterious effects of alcohol on cell function and survival and consequent organ injury may affect a number of biological processes, e.g., oxidative stress, inflammation, aberrant post-translational modifications of proteins, dysregulation in lipid metabolism, upregulation of catabolic processes and signal transduction pathways, and epigenetic changes involving DNA methylation impairments (53) (54). However, our observations may also reflect interactions between pathologies across organ systems. For example, alcohol is metabolized primarily in the liver, where it increases fatty acid synthesis(6) and leads to metabolic abnormalities that independently contribute to cardiovascular and brain pathologies(55-57). 
Our study has limitations. The observational design of UK Biobank has inherent limitations that preclude establishing causal relationships. Also, the participants are relative healthy compared to the general UK population and most are of European ancestry(58). The extent to which results are generalizable to other populations or populations of various ethnic groups needs to be explored. We relied on self-reported alcohol intake information obtained on a single occasion at the baseline assessment. This is subject to misreporting and recall bias, especially among heavy drinkers who may under-report their intake $(59,60)$ and may have led to bias in the estimation of the effects of alcohol intake on the outcomes under study.

Furthermore, the imaging data were also only available at one point in time. Future longitudinal analysis will enable effects of low or moderate alcohol intake on anatomical and structural changes of the various organs to be measured over time directly.

In summary, our findings provide new insights into the adverse effects of alcohol intake on the structures of brain, heart and aorta and on liver fat deposition. Specifically, we show that these effects are monotonic (linear-log), increasing across the full range of reported alcohol intakes in this large population sample, with no apparent threshold. Our results therefore indicate that pathological changes in major organ systems may occur at even small amounts of daily alcohol intake. This has important implications for governmental and public health policies concerning "safe" levels of alcohol drinking in the general population. Further 
research is needed, but we believe current guidelines on alcohol drinking may need to be revisited.

Disclosures E.E. acknowledges consultancy fees from OpenDNA. P.M.M. acknowledges consultancy fees from Roche, Adelphi Communications, Celgene and Biogen. He has received honoraria or speakers' honoraria from Novartis, Biogen, Medscape, Adelphi Communications and Roche and has received research or educational funds from Biogen, Novartis, GlaxoSmithKline and Nodthera. He is a member of the Scientific Advisory Board to the Board of Ipsen Pharmaceuticals.

Acknowledgements P.M.M. gratefully acknowledges generous support from Edmond J Safra Foundation and Lily Safra, the NIHR Investigator programme and the UK Dementia Research Institute, which is supported by the MRC, the Alzheimer's Society and Alzheimers Research UK. R.P. holds a fellowship supported by Rutherford Fund from Medical Research Council (MR/R0265051/1 and MR/R0265051/2). R.P. holds a fellowship supported by Rutherford Fund from Medical Research Council (MR/R0265051/1 and MR/R0265051/2). P.E. is director of the Medical Research Council (MRC) Centre for Environment and Health and acknowledges support from the MRC (MR/L01341X/1). P. E. also acknowledges support from the National Institute for Health Research (NIHR) Imperial Biomedical Research Centre and the NIHR Health Protection Research Unit in Health Impact of Environmental Hazards (HPRU-2012-10141). He is a UK Dementia Research Institute (DRI) Professor, UK DRI at Imperial College London; UK DRI is funded by the UK MRC, Alzheimer's Society, and Alzheimer's Research UK. P.E. is a co-director of the Health Data Research UK London site, which is supported, among others, by MRC, NIHR, Engineering and Physical Sciences Research Council, Economic and Social Research Council, Wellcome Trust, and British Heart Foundation. 
Role of the Funder/Sponsor: The funders had no role in the design and conduct of the study; collection, management, analysis, and interpretation of the data; preparation, review, or approval of the manuscript; and decision to submit the manuscript for publication.

Ethical approval: In UK Biobank, ethical approval for data collection was received from the North-West Multi-centre Research Ethics Committee and the research was carried out in accordance with the Declaration of Helsinki of the World Medical Association. No additional ethical approval was required for the analyses of the data

\section{References}

1. Bell S, Daskalopoulou M, Rapsomaniki E, George J, Britton A, Bobak M, et al. Association between clinically recorded alcohol consumption and initial presentation of 12 cardiovascular diseases: population based cohort study using linked health records. BMJ. 2017;356:j909.

2. GBD 2016 Alcohol Collaborators. Alcohol use and burden for 195 countries and territories, 1990-2016: a systematic analysis for the Global Burden of Disease Study 2016. Lancet. 2018;392(10152):1015-35.

3. Wood AM, Kaptoge S, Butterworth AS, Willeit P, Warnakula S, Bolton T, et al. Risk thresholds for alcohol consumption: combined analysis of individual-participant data for 599912 current drinkers in 83 prospective studies. Lancet. 2018;391(10129):1513-23.

4. Fernandez-Sola J. Cardiovascular risks and benefits of moderate and heavy alcohol consumption. Nat Rev Cardiol. 2015;12(10):576-87. 
5. Mukamal KJ, Rimm EB. Alcohol consumption: risks and benefits. Curr Atheroscler Rep. 2008;10(6):536-43.

6. Cederbaum AI. Alcohol metabolism. Clin Liver Dis. 2012;16(4):667-85.

7. Obad A, Peeran A, Little JI, Haddad GE, Tarzami ST. Alcohol-Mediated Organ Damages: Heart and Brain. Front Pharmacol. 2018;9:81.

8. Heikkinen N, Niskanen E, Kononen M, Tolmunen T, Kekkonen V, Kivimaki $\mathrm{P}$, et al. Alcohol consumption during adolescence is associated with reduced grey matter volumes. Addiction. 2017;112(4):604-13.

9. Ding J, Eigenbrodt ML, Mosley TH, Jr., Hutchinson RG, Folsom AR, Harris TB, et al. Alcohol intake and cerebral abnormalities on magnetic resonance imaging in a community-based population of middle-aged adults: the Atherosclerosis Risk in Communities (ARIC) study. Stroke. 2004;35(1):16-21.

10. Mukamal KJ, Longstreth WT, Jr., Mittleman MA, Crum RM, Siscovick DS. Alcohol consumption and subclinical findings on magnetic resonance imaging of the brain in older adults: the cardiovascular health study. Stroke. 2001;32(9):1939-46.

11. McEvoy LK, Fennema-Notestine C, Elman JA, Eyler LT, Franz CE, Hagler DJ, Jr., et al. Alcohol intake and brain white matter in middle aged men: Microscopic and macroscopic differences. Neuroimage Clin. 2018;18:390-8.

12. van Oort S, Beulens JW, van der Heijden A, Elders PJM, Stehouwer CDA, van de Luitgaarden IAT, et al. Moderate and heavy alcohol consumption are prospectively associated with decreased left ventricular ejection fraction: The Hoorn Study. Nutr Metab Cardiovasc Dis. 2020;30(1):132-40.

13. Voskoboinik A, Costello BT, La Gerche A, Prabhu S, Wong G, Flannery MD, et al. Relation of Alcohol Consumption to Left Ventricular Fibrosis Using Cardiac Magnetic Resonance Imaging. Am J Cardiol. 2019;123(3):460-5.

14. Goncalves A, Jhund PS, Claggett B, Shah AM, Konety S, Butler K, et al. Relationship between alcohol consumption and cardiac structure and function in the elderly: the Atherosclerosis Risk In Communities Study. Circ Cardiovasc Imaging. 2015;8(6).

15. Gemes K, Janszky I, Strand LB, Laszlo KD, Ahnve S, Vatten LJ, et al. Light-moderate alcohol consumption and left ventricular function among healthy, middle-aged adults: the HUNT study. BMJ Open. 2018;8(5):e020777. 
16. Sudlow C, Gallacher J, Allen N, Beral V, Burton P, Danesh J, et al. UK biobank: an open access resource for identifying the causes of a wide range of complex diseases of middle and old age. PLoS Med.

2015;12(3):e1001779.

17. Bycroft C, Freeman C, Petkova D, Band G, Elliott LT, Sharp K, et al. The UK Biobank resource with deep phenotyping and genomic data. Nature. 2018;562(7726):203-9.

18. Suzuki H, Gao H, Bai W, Evangelou E, Glocker B, O'Regan DP, et al. Abnormal brain white matter microstructure is associated with both prehypertension and hypertension. PLoS One. 2017;12(11):e0187600.

19. Evangelou E, Gao H, Chu C, Ntritsos G, Blakeley P, Butts AR, et al. New alcohol-related genes suggest shared genetic mechanisms with neuropsychiatric disorders. Nat Hum Behav. 2019;3(9):950-61.

20. Miller KL, Alfaro-Almagro F, Bangerter NK, Thomas DL, Yacoub E, Xu J, et al. Multimodal population brain imaging in the UK Biobank prospective epidemiological study. Nat Neurosci. 2016;19(11):1523-36.

21. Good CD, Johnsrude IS, Ashburner J, Henson RN, Friston KJ, Frackowiak RS. A voxel-based morphometric study of ageing in 465 normal adult human brains. Neuroimage. 2001;14(1 Pt 1):21-36.

22. Elliott LT, Sharp K, Alfaro-Almagro F, Shi S, Miller KL, Douaud G, et al. Genome-wide association studies of brain imaging phenotypes in UK Biobank. Nature. 2018;562(7726):210-6.

23. Zhang H, Schneider T, Wheeler-Kingshott CA, Alexander DC. NODDI: practical in vivo neurite orientation dispersion and density imaging of the human brain. Neuroimage. 2012;61(4):1000-16.

24. Available from https://www.ukbiobank.ac.uk/data-showcase/. Last accessed on 16/09/2020.

25. Petersen SE, Matthews PM, Francis JM, Robson MD, Zemrak F, Boubertakh R, et al. UK Biobank's cardiovascular magnetic resonance protocol. J Cardiovasc Magn Reson. 2016;18:8.

26. Bai W, Sinclair M, Tarroni G, Oktay O, Rajchl M, Vaillant G, et al. Automated cardiovascular magnetic resonance image analysis with fully convolutional networks. J Cardiovasc Magn Reson. 2018;20(1):65.

27. Wenjia Bai HS, Chen Qin, Giacomo Tarroni, Ozan Oktay, Paul M. Matthews, Danile Rueckert. Reccurent neural networks for aortic image sequence segmentation with sparse annotations. In: A. Frangi JS, C. Davatzikos, C. Alberola-Lopez, G Fichtinger, ed. MICCAI 2018: Springer; 2018. 
28. Wilman HR, Kelly M, Garratt S, Matthews PM, Milanesi M, Herlihy A, et al. Characterisation of liver fat in the UK Biobank cohort. PLoS One. 2017;12(2):e0172921.

29. Enzinger C, Fazekas F, Matthews PM, Ropele S, Schmidt H, Smith S, et al. Risk factors for progression of brain atrophy in aging: six-year follow ${ }^{-}$ up of normal subjects. Neurology. 2005;64(10):1704-11.

30. Mollink J, Kleinnijenhuis M, Cappellen van Walsum AV, Sotiropoulos SN, Cottaar M, Mirfin C, et al. Evaluating fibre orientation dispersion in white matter: Comparison of diffusion MRI, histology and polarized light imaging. Neuroimage. 2017;157:561-74.

31. UK Chief Medical Officers' Low Risk Drinking Guidelines. 2016.

32. U.S. Department of Health and Human Services and U.S. Department of Agriculture. 2015-2020 Dietary Guidelines for Americans. 2015;8th Ediction.

33. Cosa A, Moreno A, Pacheco-Torres J, Ciccocioppo R, Hyytia P, Sommer WH, et al. Multi-modal MRI classifiers identify excessive alcohol consumption and treatment effects in the brain. Addict Biol. 2017;22(5):1459-72.

34. Topiwala A, Allan CL, Valkanova V, Zsoldos E, Filippini N, Sexton C, et al. Moderate alcohol consumption as risk factor for adverse brain outcomes and cognitive decline: longitudinal cohort study. BMJ. 2017;357:j2353.

35. Ferizi U, Scherrer B, Schneider T, Alipoor M, Eufracio O, Fick RHJ, et al. Diffusion MRI microstructure models with in vivo human brain Connectome data: results from a multi-group comparison. NMR Biomed. 2017;30(9).

36. Mole JP, Subramanian L, Bracht T, Morris H, Metzler-Baddeley C, Linden DE. Increased fractional anisotropy in the motor tracts of Parkinson's disease suggests compensatory neuroplasticity or selective neurodegeneration. Eur Radiol. 2016;26(10):3327-35.

37. Douaud G, Jbabdi S, Behrens TE, Menke RA, Gass A, Monsch AU, et al. DTI measures in crossing-fibre areas: increased diffusion anisotropy reveals early white matter alteration in MCI and mild Alzheimer's disease. Neuroimage. 2011;55(3):880-90.

38. Manolio TA, Levy D, Garrison RJ, Castelli WP, Kannel WB. Relation of alcohol intake to left ventricular mass: The Framingham Study. J Am Coll Cardiol. 1991;17(3):717-21. 
39. Levy D, Garrison RJ, Savage DD, Kannel WB, Castelli WP. Prognostic implications of echocardiographically determined left ventricular mass in the Framingham Heart Study. N Engl J Med. 1990;322(22):1561-6.

40. Benjamin EJ, D'Agostino RB, Belanger AJ, Wolf PA, Levy D. Left atrial size and the risk of stroke and death. The Framingham Heart Study. Circulation. 1995;92(4):835-41.

41. Gottdiener JS, Kitzman DW, Aurigemma GP, Arnold AM, Manolio TA. Left atrial volume, geometry, and function in systolic and diastolic heart failure of persons $>$ or $=65$ years of age (the cardiovascular health study). Am J Cardiol. 2006;97(1):83-9.

42. Erbel R, Eggebrecht H. Aortic dimensions and the risk of dissection. Heart. 2006;92(1):137-42.

43. Redheuil A, Wu CO, Kachenoura N, Ohyama Y, Yan RT, Bertoni AG, et al. Proximal aortic distensibility is an independent predictor of all-cause mortality and incident CV events: the MESA study. J Am Coll Cardiol. 2014;64(24):2619-29.

44. Marmot MG, Rose G, Shipley MJ, Thomas BJ. Alcohol and mortality: a Ushaped curve. Lancet. 1981;1(8220 Pt 1):580-3.

45. Bellentani S, Saccoccio G, Costa G, Tiribelli C, Manenti F, Sodde M, et al. Drinking habits as cofactors of risk for alcohol induced liver damage. The Dionysos Study Group. Gut. 1997;41(6):845-50.

46. Moriya A, Iwasaki Y, Ohguchi S, Kayashima E, Mitsumune T, Taniguchi $\mathrm{H}$, et al. Roles of alcohol consumption in fatty liver: a longitudinal study. $\mathrm{J}$ Hepatol. 2015;62(4):921-7.

47. Hashimoto Y, Hamaguchi M, Kojima T, Ohshima Y, Ohbora A, Kato T, et al. Modest alcohol consumption reduces the incidence of fatty liver in men: a population-based large-scale cohort study. J Gastroenterol Hepatol. 2015;30(3):546-52.

48. Yamada T, Fukatsu M, Suzuki S, Yoshida T, Tokudome S, Joh T. Alcohol drinking may not be a major risk factor for fatty liver in Japanese undergoing a health checkup. Dig Dis Sci. 2010;55(1):176-82.

49. Kechagias S, Zanjani S, Gjellan S, Leinhard OD, Kihlberg J, Smedby O, et al. Effects of moderate red wine consumption on liver fat and blood lipids: a prospective randomized study. Ann Med. 2011;43(7):545-54.

50. van Eekelen E, Beulens JWJ, Geelen A, Schrauwen-Hinderling VB, Lamb $\mathrm{H}$, de Roos A, et al. Consumption of Alcoholic and Sugar-Sweetened Beverages is Associated with Increased Liver Fat Content in Middle-Aged Men and Women. J Nutr. 2019;149(4):649-58. 
51. Linge J, Whitcher B, Borga M, Dahlqvist Leinhard O. Sub-phenotyping Metabolic Disorders Using Body Composition: An Individualized, Nonparametric Approach Utilizing Large Data Sets. Obesity (Silver Spring). 2019;27(7):1190-9.

52. Goncalves A, Claggett B, Jhund PS, Rosamond W, Deswal A, Aguilar D, et al. Alcohol consumption and risk of heart failure: the Atherosclerosis Risk in Communities Study. Eur Heart J. 2015;36(15):939-45.

53. Souza-Smith FM, Lang CH, Nagy LE, Bailey SM, Parsons LH, Murray GJ. Physiological processes underlying organ injury in alcohol abuse. Am J Physiol Endocrinol Metab. 2016;311(3):E605-19.

54. Osna NA, Kharbanda KK. Multi-Organ Alcohol-Related Damage: Mechanisms and Treatment. Biomolecules. 2016;6(2).

55. Suzuki H, Venkataraman AV, Bai W, Guitton F, Guo Y, Dehghan A, et al. Associations of Regional Brain Structural Differences With Aging, Modifiable Risk Factors for Dementia, and Cognitive Performance. JAMA Netw Open. 2019;2(12):e1917257.

56. Wilson PW, D'Agostino RB, Parise H, Sullivan L, Meigs JB. Metabolic syndrome as a precursor of cardiovascular disease and type 2 diabetes mellitus. Circulation. 2005;112(20):3066-72.

57. Cai H, Cong WN, Ji S, Rothman S, Maudsley S, Martin B. Metabolic dysfunction in Alzheimer's disease and related neurodegenerative disorders. Curr Alzheimer Res. 2012;9(1):5-17.

58. Fry A, Littlejohns TJ, Sudlow C, Doherty N, Adamska L, Sprosen T, et al. Comparison of Sociodemographic and Health-Related Characteristics of UK Biobank Participants With Those of the General Population. Am J Epidemiol. 2017;186(9):1026-34.

59. Boniface S, Kneale J, Shelton N. Drinking pattern is more strongly associated with under-reporting of alcohol consumption than sociodemographic factors: evidence from a mixed-methods study. BMC Public Health. 2014;14:1297.

60. Greenfield TK, Kerr WC. Alcohol measurement methodology in epidemiology: recent advances and opportunities. Addiction. 2008;103(7):1082-99. 
medRxiv preprint doi: https://doi.org/10.1101/2021.01.20.21249931; this version posted January 20, 2021. The copyright holder for this preprint (which was not certified by peer review) is the author/funder, who has granted medRxiv a license to display the preprint in perpetuity. It is made available under a CC-BY-ND 4.0 International license.

Table 1. Participant characteristics

\begin{tabular}{lccccc}
\hline & $\begin{array}{c}\text { Brain grey } \\
\text { matter }\end{array}$ & $\begin{array}{c}\text { Brain white } \\
\text { matter }\end{array}$ & Heart & Aorta & Liver \\
\hline $\mathrm{N}$ & 10143 & 9053 & 11821 & 12376 & 3649 \\
Baseline characteristics & & & & & \\
Age (yrs) (Mean $\pm \mathrm{SD})$ & $62.9 \pm 7.4$ & $62.9 \pm 7.4$ & $62.8 \pm 7.4$ & $63.0 \pm 7.4$ & $55.7 \pm 7.5$ \\
Male (\%) & 49.8 & 49.4 & 49.9 & 50.2 & 50.5 \\
Caucasian (\%) & 99.8 & 99.8 & 99.8 & 99.8 & 93.2 \\
Educational attainment (\%) & 53.2 & 53.5 & 53.5 & 52.9 & 53.1 \\
Body mass index (Mean \pm SD) & $26.7 \pm 4.4$ & $26.6 \pm 4.3$ & $26.5 \pm 4.3$ & $26.5 \pm 4.2$ & $26.5 \pm 4.1$ \\
Hypertension (\%) & 39.6 & 39.3 & 39.0 & 39.1 & 46.5 \\
Diabetes (\%) & 5.2 & 5.0 & 4.7 & 4.9 & 2.5 \\
Smoking history (\%) & 39.7 & 39.4 & 38.6 & 39.1 & 36 \\
Alcohol consumption (g/d) (Median-IQR) & 14.29 & 14.29 & 14.29 & 14.29 & 16.61 \\
\hline
\end{tabular}


Table 2. Structural imaging phenotypes for brain $(\mathrm{N}=10,143)$, heart $(\mathrm{N}=11,821)$ and aorta $(\mathrm{N}=12,376)$ in the UK Biobank

\begin{tabular}{|c|c|}
\hline Imaging derived phenotypes & Mean \pm SD \\
\hline \multicolumn{2}{|l|}{ Brain } \\
\hline Normalised brain volume & $0.72 \pm 0.045$ \\
\hline Normalised grey matter volume & $0.43 \pm 0.034$ \\
\hline Normalised white matter volume & $0.29 \pm 0.020$ \\
\hline \multicolumn{2}{|l|}{ Heart } \\
\hline Left ventricular mass index $\left(\mathrm{g} / \mathrm{m}^{2}\right)$ & $46.2 \pm 8.5$ \\
\hline Left ventricular end-diastolic volume index $\left(\mathrm{ml} / \mathrm{m}^{2}\right)$ & $80.0 \pm 13.7$ \\
\hline Left ventricular ejection fraction (\%) & $59.6 \pm 5.9$ \\
\hline Left atrial volume index $\left(\mathrm{ml} / \mathrm{m}^{2}\right)$ & $38.8 \pm 10.7$ \\
\hline Right ventricular end-diastolic volume index $\left(\mathrm{ml} / \mathrm{m}^{2}\right)$ & $84.5 \pm 15.4$ \\
\hline Right ventricular ejection fraction (\%) & $57.3 \pm 6.0$ \\
\hline Right atrial volume index $\left(\mathrm{ml} / \mathrm{m}^{2}\right)$ & $46.1 \pm 13.1$ \\
\hline \multicolumn{2}{|l|}{ Aorta } \\
\hline Ascending aortic area index $\left(\mathrm{mm}^{2} / \mathrm{m}^{2}\right)$ & $455.3 \pm 91.9$ \\
\hline Ascending aortic distensibility $\left(10^{-3} \mathrm{mmHg}^{-1}\right)$ & $1.98 \pm 1.17$ \\
\hline Descending aortic area index $\left(\mathrm{mm}^{2} / \mathrm{m}^{2}\right)$ & $254.1 \pm 43.3$ \\
\hline Descending aortic distensibility $\left(10^{-3} \mathrm{mmHg}^{-1}\right)$ & $2.64 \pm 1.25$ \\
\hline
\end{tabular}

SD: Standard Deviation 
Table 3. Associations of age and alcohol consumption with cardiac $(\mathrm{N}=11,821)$ and aortic $(\mathrm{N}=12,376)$ imaging phenotypes

\begin{tabular}{|c|c|c|c|c|}
\hline & Aging/ year, estimate* $\pm \mathrm{SE}$ & P-value & Alcohol, estimate* \pm SE & P-value \\
\hline \multicolumn{5}{|c|}{ 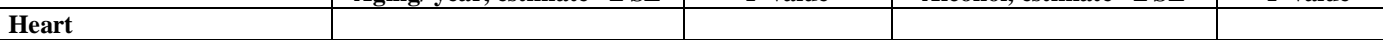 } \\
\hline Left ventricular mass index & $-0.10 \pm 0.01$ & $1.3 \times 10^{-31}$ & $0.36 \pm 0.04$ & $8.5 \times 10^{-22}$ \\
\hline $\begin{array}{l}\text { Left ventricular end- } \\
\text { diastolic volume index }\end{array}$ & $-0.33 \pm 0.02$ & $6.0 \times 10^{-90}$ & $0.61 \pm 0.07$ & $4.1 \times 10^{-17}$ \\
\hline $\begin{array}{l}\text { Left ventricular ejection } \\
\text { fraction }(\%)\end{array}$ & $0.04 \pm 0.01$ & $6.3 \times 10^{-7}$ & $0.05 \pm 0.03$ & 0.16 \\
\hline $\begin{array}{l}\text { Left atrial volume index } \\
\left(\mathrm{ml} / \mathrm{m}^{2}\right)\end{array}$ & $-0.12 \pm 0.01$ & $8.9 \times 10^{-17}$ & $0.43 \pm 0.06$ & $6.0 \times 10^{-12}$ \\
\hline $\begin{array}{l}\text { Right ventricular end- } \\
\text { diastolic volume index } \\
\left(\mathrm{ml} / \mathrm{m}^{2}\right)\end{array}$ & $-0.33 \pm 0.02$ & $5.6 \times 10^{-80}$ & $0.57 \pm 0.08$ & $2.5 \times 10^{-13}$ \\
\hline $\begin{array}{l}\text { Right ventricular ejection } \\
\text { fraction }(\%)\end{array}$ & $0.04 \pm 0.01$ & $1.6 \times 10^{-8}$ & $0.05 \pm 0.03$ & 0.13 \\
\hline $\begin{array}{l}\text { Right atrial volume index } \\
\left(\mathrm{ml} / \mathrm{m}^{2}\right)\end{array}$ & $0.04 \pm 0.02$ & $6.9 \times 10^{-3}$ & $0.26 \pm 0.07$ & $3.0 \times 10^{-4}$ \\
\hline \multicolumn{5}{|l|}{ Aorta } \\
\hline $\begin{array}{l}\text { Ascending aortic area index } \\
\left(\mathrm{mm}^{2} / \mathrm{m}^{2}\right)\end{array}$ & $2.75 \pm 0.11$ & $4.0 \times 10^{-130}$ & $2.64 \pm 0.50$ & $1.5 \times 10^{-7}$ \\
\hline $\begin{array}{l}\text { Ascending aortic } \\
\text { distensibility }\left(10^{-3} \mathrm{mmHg}^{-1}\right)\end{array}$ & $-0.09 \pm 0.001$ & $<1 \times 10^{-300}$ & $-0.006 \pm 0.005$ & 0.22 \\
\hline $\begin{array}{l}\text { Descending aortic area } \\
\text { index }\left(\mathrm{mm}^{2} / \mathrm{m}^{2}\right)\end{array}$ & $1.86 \pm 0.05$ & $2.0 \times 10^{-291}$ & $1.34 \pm 0.22$ & $2.1 \times 10^{-9}$ \\
\hline $\begin{array}{l}\text { Descending aortic } \\
\text { distensibility }\left(10^{-3} \mathrm{mmHg}^{-1}\right)\end{array}$ & $-0.09 \pm 0.001$ & $<1 \times 10^{-300}$ & $-0.02 \pm 0.005$ & $7.5 \times 10^{-4}$ \\
\hline
\end{tabular}

*Estimates \pm SE define coefficients for cardiac and aortic imaging phenotype changes per year age or per doubling of alcohol consumption $(\mathrm{g} / \mathrm{d})$ with their standard errors. The aging model was adjusted for sex, ethnicity, body mass index, and prevalence of college degree education, hypertension, diabetes, and smoking history. The alcohol consumption model was adjusted for age, sex, ethnicity, body mass index, and prevalence of college degree education, hypertension, diabetes, and smoking history. Abbreviation: SE, standard error. 
medRxiv preprint doi: https://doi.org/10.1101/2021.01.20.21249931; this version posted January 20, 2021. The copyright holder for this preprint (which was not certified by peer review) is the author/funder, who has granted medRxiv a license to display the preprint in perpetuity.

It is made available under a CC-BY-ND 4.0 International license.

\section{Figure 1. Voxel-wise associations of alcohol consumption with brain grey matter} concentrations $(\mathbf{N}=\mathbf{1 0 , 1 4 3})$. Highlighted clusters define regions in which reductions of spatially-normalised grey matter concentration (an indirect measure of relative tissue density) are inversely correlated with $\log _{2}$-transformed alcohol consumption $(\mathrm{g} / \mathrm{d})$. The analysis suggests higher relative atrophy in the cingulate cortex (light blue arrowheads, panel A and B), thalamus (green arrowheads, panel A and C), orbital frontal cortex (red arrowheads, panel A and E), insular cortex (dark blue arrowheads, panels D and E). Broken lines in panel A show levels for axial images in panels B-D. The voxel-wise parametric model was adjusted for age, sex, ethnicity, body mass index, college degree education, hypertension, diabetes, and smoking history. The results are displayed on the MRI template available in SPM12 at axial slices of $46.5 \mathrm{~mm}(\mathrm{~B}), 22.5 \mathrm{~mm}(\mathrm{C}), 6 \mathrm{~mm}(\mathrm{D}),-7.5 \mathrm{~mm}(\mathrm{E})$ and $-21 \mathrm{~mm}(\mathrm{~F})$ relative to the bregma. The calibration bar provides the colour range use to describe t-scores calculated using a family-wise error (FWE)-corrected threshold of $P<0.05$.

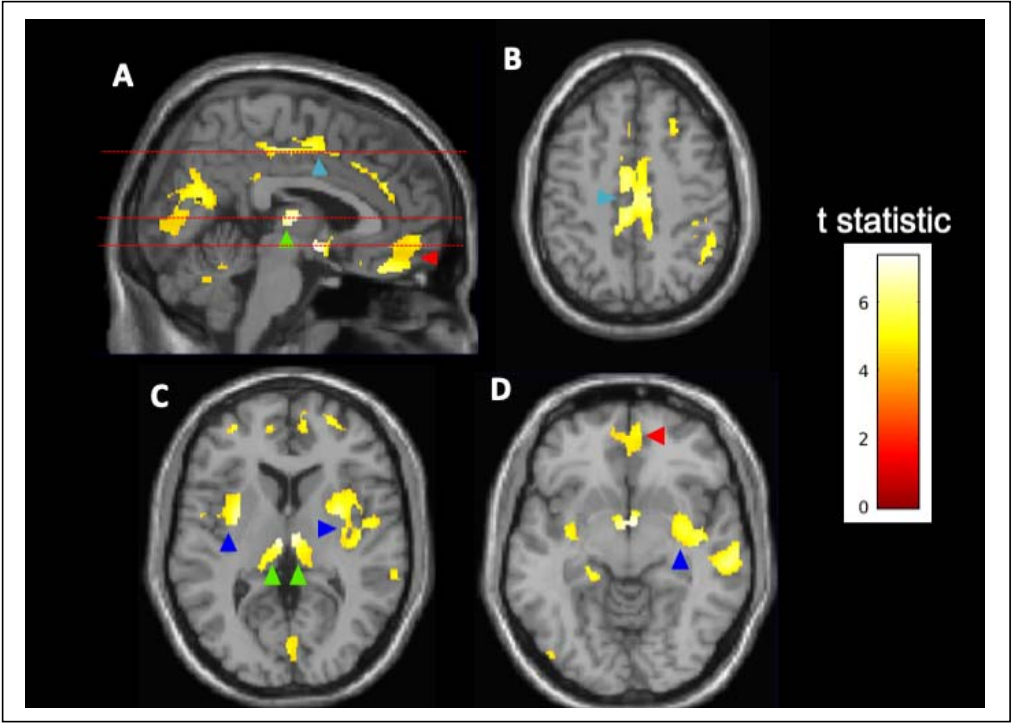

